\title{
Genomics approach to the analysis of bacterial communities dynamics in Hirschsprung's disease-associated enterocolitis: a pilot study
}

\author{
C. De Filippo - A. Pini-Prato - G. Mattioli - S. Avanzini - G. Rapuzzi • \\ D. Cavalieri - M. Di Paola • I. Stefanini - I. Ceccherini - D. Mavilio • \\ P. Lionetti • V. Jasonni
}

Accepted: 2 March 2010/Published online: 20 March 2010

(C) Springer-Verlag 2010

\begin{abstract}
Introduction The most invalidating and life-threatening complication in Hirschsprung's disease patients (HSCR) is Hirschsprung's disease-associated enterocolitis (HAEC). The mechanisms underlying enterocolitis have not been identified. The limited knowledge of the role of intestinal microflora is in part due to the complexity of the intestinal microbiome and to the limitation of cultivation-based technologies, given that less than $25 \%$ of the intestinal bacterial species can be cultured.

Materials and methods We used amplified ribosomal DNA restriction analysis (ARDRA) with four different restriction enzymes to study variations of microflora
\end{abstract}

C. De Filippo - D. Cavalieri - M. Di Paola - I. Stefanini Department of Pharmacology, University of Florence, Florence, Italy

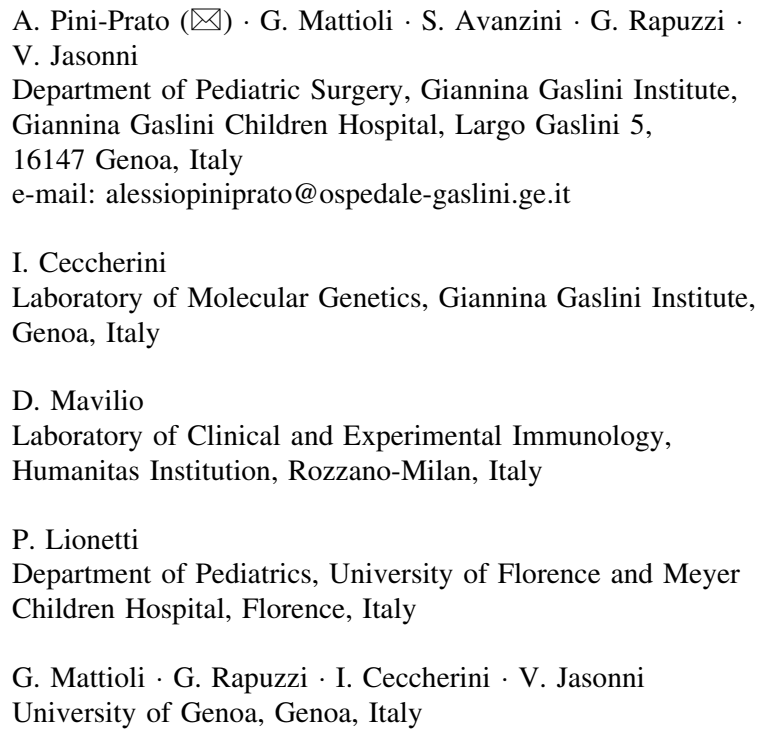

composition of the stools of a selected HSCR patient in different clinical conditions (acute phase vs. remission). Results We assessed a total of 15 stool specimens belonging to the same 3-year-old male patient suffering from HSCR, which were harvested during 4 HAEC episodes and remission phases. Restriction analysis showed that HAEC episodes seem to cluster together at ARDRA analysis, thus suggesting a sort of predisposing bacterial community for HAEC development and the need for a microflora equilibrium to maintain wellness.

Conclusions This approach proved to be effective, useful and powerful in assessing microflora dynamics and indicated that the differences in microflora associated with acute HAEC or remission are likely to result from a combination of disease activity and different antibiotic therapies. ARDRA proved to be useful in discriminating disease versus remission. Our findings indicated that HAEC results from a change in the equilibrium between bacterial species or from altered discrimination of harmless from harmful microorganisms, challenging the definition of pathogenic and non-pathogenic species. Based on these results, we propose ARDRA as a rapid inexpensive tool to assess microflora dynamics during HAEC episodes.

Keywords Hirschsprung - Enterocolitis - Microbioma · Genome · Diagnosis

\section{Introduction}

Hirschsprung's disease (HSCR) is a developmental disorder of the enteric nervous system characterized by the congenital absence of parasympathetic intrinsic ganglion cells in the submucosal and myenteric plexuses of the hindgut with variable proximal extension. Clinical features 
of HSCR are heterogeneous: patients can experience neonatal intestinal obstruction, delayed meconium passage, chronic constipation, abdominal distension and the most debilitating and life-threatening complication, named HSCR-associated enterocolitis (HAEC) [1-3]. HAEC is defined as a clinical condition characterized by explosive diarrhea, abdominal distension, fever and impending septic shock [4].

HAEC can occur at any time starting from the neonatal period and is often independent of surgical treatment, though its severity seems higher before surgery [5]. The incidence of HAEC ranges from 20 to $58 \%$ and its mortality rate has significantly decreased over the past 20 years from 30 to $1 \%$ due to early diagnosis, rectal decompression, appropriate vigorous resuscitation and antibiotic therapy. However, a number of patients with HAEC need an enterostomy (colostomy or ileostomy). Even though several hypotheses have been formulated with regard to the physiopathology of HAEC, the concurring mechanisms underlying enterocolitis have not been identified yet. Histological and immunological studies suggest that some patients are prone to recurrent HAEC due either to persistent inflammation within the bowel or to immunodeficiency/dysfunctions of cells of the immune system [6]. As it has been observed for inflammatory bowel diseases (IBD), the role of intestinal microflora is indeed of paramount importance in determining the onset and the gravity of HAEC [7]. The intestinal immune system maintains immunologic homeostasis against gut luminal antigens. Alternatively, it also provides an appropriate immune response against microbial pathogens at the local site of inflammation [8-10]. Clostridium difficile and Rotavirus, have been associated with HAEC, although the etiology is extremely variable [11] and there are also reports of the presence of different Candida species [12]. The limited knowledge of the HAEC determinants is in part also due to the complexity of the intestinal microbiome. One genomic estimate of the biodiversity of intestinal microflora indicated roughly 400 different phylotypes that can colonize both intestinal lumen and mucosa, being less than $20 \%$ cultivable [13]. The traditional methods based on culturing bacteria require fresh material and considerable experimental equipment for anaerobic culturing, which is difficult to undertake in the field. However, culture-independent methodologies based on the amplification of bacterial $16 \mathrm{~S}$ rRNA genes by polymerase chain reaction (PCR) require only bacterial genomic DNA as templates. The $16 \mathrm{~S}$ rDNA region is universally considered a good marker to screen and identify bacterial species due to the variability of this region. To assess the structure of a particular microbial community, the amplified ribosomal DNA can be analyzed by amplified ribosomal DNA restriction analysis (ARDRA), denaturing gradient gel electrophoresis (DGGE) or temperature gradient gel electrophoresis (TGGE) [14].

Many authors use $16 \mathrm{~S}$ rDNA restriction analysis to characterize bacterial species isolated from nature. The vast majority of these studies are performed following cultivation and isolation in pure culture of a bacterial species. In a sizeable fraction of the cited literature, ARDRA was not performed directly on PCR-amplified community DNA, but rather on clones from a library of PCR-amplified DNA [15, 16]. In these types of experiments, ARDRA is used to identify major groups of species from among the clones by patterns of similarity in their restriction fragments. Sequencing is then done on representative clones from each ARDRA-defined group. In this way, ARDRA is used as a shortcut to minimize sequencing of clone libraries.

The aim of this study was to explore the potential of ARDRA technique to assess changes in microbial composition of the stool of HSCR patients. In particular, we aimed at assessing whether DNA-based technologies can overcome the limitations derived from limited cultivability of bacterial species.

\section{Methods}

Fresh stools were collected from a 3-year-old male patient with an ultra-long form of HSCR (total colonic aganglionosis, TCSA) during different enterocolitis and remission episodes between June and October 2008 (Table 1). The diagnosis of intestinal aganglionosis was obtained with histochemistry on rectal suction biopsies according to widely accepted criteria [1]. The diagnosis was confirmed with intraoperative histochemistry and pathological analysis of the resected bowel. Enterocolitis was diagnosed according to the criteria described by Pastor AC et al. in 2009 [29].

Furthermore, the patient's DNA underwent molecular analysis of RET proto-oncogene, as previously reported [18, 19], without finding any mutation. Extensive genotyping of the same gene revealed a homozygous condition for an SNP allele (RET + 3_T allele), known to be a predisposing factor for HSCR development [20].

\section{Ethical approval}

The Giannina Gaslini Institute Ethic Committee Board approved this study as a part of a comprehensive research on Hirschsprung's disease-associated enterocolitis. A detailed informed consent was obtained by the parents before starting with this study. 
Table 1 Summary of fecal samples obtained from a single 3 -year-old pediatric patient affected by HSCR with recurrent enterocolitis episodes between June and October 2008

\begin{tabular}{lll}
\hline Sample's ID & Enterocolitis phases & Antibiotic treatment \\
\hline Period 1 & & \\
T0 & Pre-enterocolitis & 12 h after stopping amoxicillin/clavulanic acid (RD) \\
T1 & Acute enterocolitis & Metronidazole \\
T2 & Partial remission & Amoxicillin/clavulanic acid (RD) \\
T3 & Partial remission & Amoxicillin/clavulanic acid (RD) \\
T4 & Remission & Amoxicillin/clavulanic acid (RD) \\
Period 2 & & \\
T5 & Remission & Amoxicillin/clavulanic acid (RD) \\
T6 & Pre-enterocolitis & Amoxicillin/clavulanic acid (RD) \\
T7 & Acute enterocolitis & Metronidazole \\
Period 3 & & \\
T8 & Remission & Amoxicillin/clavulanic acid (RD) \\
T9 & Pre-enterocolitis & Amoxicillin/clavulanic acid \\
T10 & Pre-remission & Amoxicillin/clavulanic acid \\
T11 & Pre-remission & Amoxicillin/clavulanic acid \\
Period 4 & & \\
T12 & Acute enterocolitis & Metronidazole \\
T13 & Acute enterocolitis & Metronidazole \\
T14 & Partial remission & Amoxicillin/clavulanic acid \\
T15 & Remission & Amoxicillin/clavulanic acid $^{\mathrm{a}}$ \\
\hline
\end{tabular}

Collection of fecal samples and DNA extraction

Stool samples were kept at $4^{\circ} \mathrm{C}$ until the analysis was performed. After homogenization by vigorous shaking by hand, a portion of the suspension was centrifuged $(10,000 \mathrm{~g}$, for $10 \mathrm{~min}$ at $4^{\circ} \mathrm{C}$ ) to collect pellets. The pellets were subjected to the DNA extraction procedure according to Zoetendal et al. [17].

\section{S rDNA PCR amplification}

Universal primers (forward primer Bact8F, 5'-AGAG TTTGATCCTGGCTCAG-3' and a reverse primer Bact1510, 5'-CGGTTACCTTGTTACGACTT-3') were used to amplify the bacterial $16 \mathrm{~S}$ rDNA. PCRs were performed with PlatinumTaq DNA polymerase (Invitrogen, Milano, Italy).

Each $50 \mathrm{ml}$ PCR mixture contained $20 \mathrm{mM}$ Tris- $\mathrm{HCl}(\mathrm{pH}$ 8.4), $50 \mathrm{mM} \mathrm{KCl}, 1.5 \mathrm{mM} \mathrm{MgCl}_{2}, 0.25 \mathrm{mM}$ each of deoxynucleoside triphosphate (dNTP), $2.5 \mathrm{U}$ Taq DNA polymerase, $0.4 \mu \mathrm{M}$ of each primer and $100 \mathrm{ng}$ of genomic bacterial DNA. The samples were amplified in a BIO-016, MJ Research PCR machine using the following program: initial denaturation at $94^{\circ} \mathrm{C}$ for $2 \mathrm{~min} ; 35$ thermal cycles of $94^{\circ} \mathrm{C}$ for $30 \mathrm{~s}, 60^{\circ} \mathrm{C}$ for $30 \mathrm{~s}$ and $72^{\circ} \mathrm{C}$ for $1 \mathrm{~min}$; and final elongation at $72^{\circ} \mathrm{C}$ for $8 \mathrm{~min}$. The size and amount of the PCR products were confirmed by analyzing $5 \mu \mathrm{l}$ samples by $1 \%$ agarose gel (wt/vol) electrophoresis and ethidium bromide staining. All samples were subjected to ARDRA analysis.

\section{ARDRA technique}

We did set up efficient bacterial genomic DNA extraction techniques from stools to amplify $16 \mathrm{~S}$ rDNA from all samples using selected universal primers. We initially used ten different restriction enzymes that were indicated as diagnostic [21, 22] to digest one sample of amplification pool of bacterial $16 \mathrm{~S}$ rDNA according to the method of Vaneechoutte et al. [23]. The pools of digested DNA were analyzed and, based on the results, we selected four $4 \mathrm{bp}$ restriction enzymes (HaeIII, AluI, MspI, RsaI-Fermentas, Cornaredo, Milan, Italy) that generated more diagnostic restriction profiles, producing the greatest number of restriction fragments in our samples (Fig. 1a, b, c, d).

As much as $10 \mu \mathrm{l}$ of $16 \mathrm{~S}$ rDNA PCR product was digested with $5 \mathrm{U}$ of the previously indicated restriction enzymes for $3 \mathrm{~h}$ at $37^{\circ} \mathrm{C}$. The restriction fragments were separated on a $2.5 \%$ agarose gel for $2 \mathrm{~h}$ at $80 \mathrm{~V}$ and visualized using Image Quant 350 gel documentation system (GE Healthcare, Amersham Biosciences Cologno Monzese, Italy). A 123 bp ladder (Invitrogen, Milano, Italy) was used as a DNA marker. By way of illustration, a dendrogram, based on ARDRA patterns, was constructed using the PHYLIP program (Phylogeny Interference Package-version 3.6 Dept of Genome Sciences, University of Washington, Seattle, http://evolution.genetics.washington. edu/phylip/getme.html) with $1 \%$ tolerance (i.e. bands that differ by about seven nucleotides or less are considered identical) and taking only fragments from 80 to 1,000 

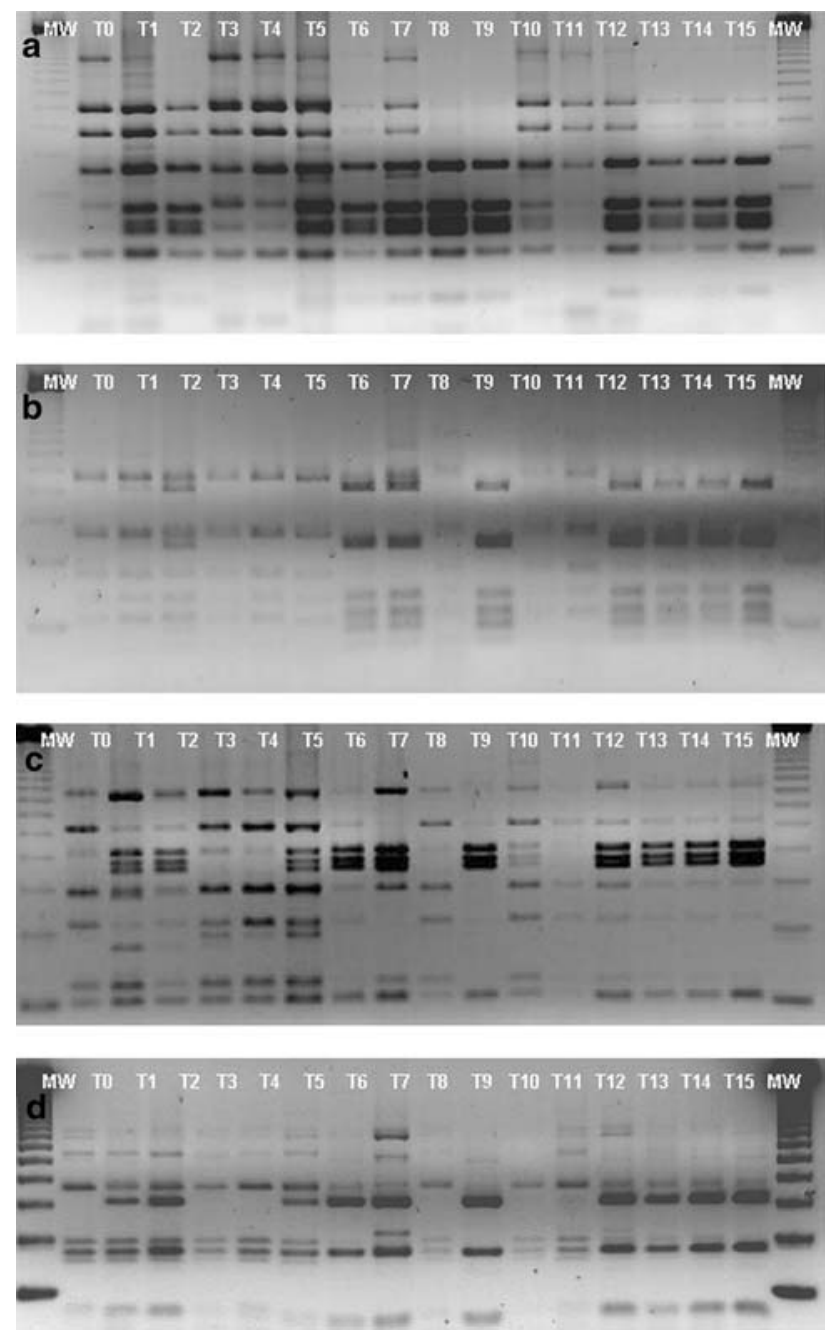

Fig. 1 Gel image of ARDRA analysis of bacterial 16S rDNA retrieved from the feces of an HSCR patient in different phases of the disease (HAEC vs. remission phase) using, respectively, HaeIII (a), MspI (b), RsaI (c) and AluI (d). The sample's IDs are reported on Table 1. $M W$ ladder $123 \mathrm{bp}$. The detection of the band was performed automatically on the original image using the Image Quant 350 gel documentation system (GE Healthcare, Amersham Biosciences) for the hierarchical clustering analysis

nucleotides into account with the same methodology used to compare restriction patterns from single bacterial species isolates [24].

\section{Results}

Patient clinical features and enterocolitis episodes

During the study period (see Table 1 for details), the patient underwent prolonged and repetitive treatments with oral amoxicillin/clavulanic acid at a dose of $40 \mathrm{mg} /$ $\mathrm{kg}$ per die for amoxicillin and $28.5 \mathrm{mg} / \mathrm{kg}$ per die for clavulanic acid, to decontaminate the bowel and reduce fecal output. The first event of enterocolitis occurred after amoxicillin/clavulanic acid discontinuation (sample T0 was collected $12 \mathrm{~h}$ after antibiotic suspension), which was attempted to re-establish a native and natural bacterial flora by antibiotics cycling. The patient was then treated with oral metronidazole at a dosage of $20 \mathrm{mg} / \mathrm{kg}$ per die, but the child developed enterocolitis (T1). Further remission was obtained on re-introducing amoxicillin/ clavulanic acid therapy (T2-T5). During the second episode of enterocolitis, the patient was administrated oral metronidazole ( $200 \mathrm{mg} / \mathrm{die})$, according to widely accepted guidelines $[4,5]$, to treat a presumable anaerobic microflora overgrowth (T6), but he worsened (T7). A long remission phase (T8) was subsequently experienced after metronidazole discontinuation. A third episode was anticipated by a pre-enterocolitis phase (T9), which was preventively treated by doubling the dose of amoxicillin/ clavulanic acid (T10-T11) avoiding a real enterocolitis recurrence. A fourth enterocolitis episode occurred (T12-T13), but also in this occasion metronidazole treatment worsened clinical conditions. In this latter event, remission was obtained by doubling the dose of amoxicillin/ clavulanic acid (T14-T15). All stool samples were initially characterized by conventional microbiological approaches. Cultivation-based techniques and immunohistochemical techniques excluded the presence of pathogens such as Salmonella, Shigella, Campylobacter and C. difficile. Furthermore, those techniques were not able to discriminate between enterocolitis or remission phases based on the presence of characteristic cultivable bacteria (data not shown).

\section{Analysis of restriction profiles}

The clustering of the 16S rDNA restriction profiles (Fig. 2) on the mixed bacterial populations allowed discriminating every sample as unique and, in particular, to assess sample similarity according to a well-established methodology. In particular, we could observe that the enterocolitis samples corresponded to specific changes in microflora with the onset of enterocolitis episodes.

The first enterocolitis episode (Fig. 2) showed coherence in the order of the samples: the samples clustered temporally together and the order was in accordance with disease progression, from the pre-enterocolitis phase to acute enterocolitis and remission. The first episode followed suspension of antibiotic treatment with amoxicillin/ clavulanic acid. The pre-enterocolitis phase in this patient represented the starting point of microbial dynamics that developed during the following months, as it was different from all the others and behaved like a "root" of a clustering tree. The acute enterocolitis episodes named second and third were closer to each other compared to the first 


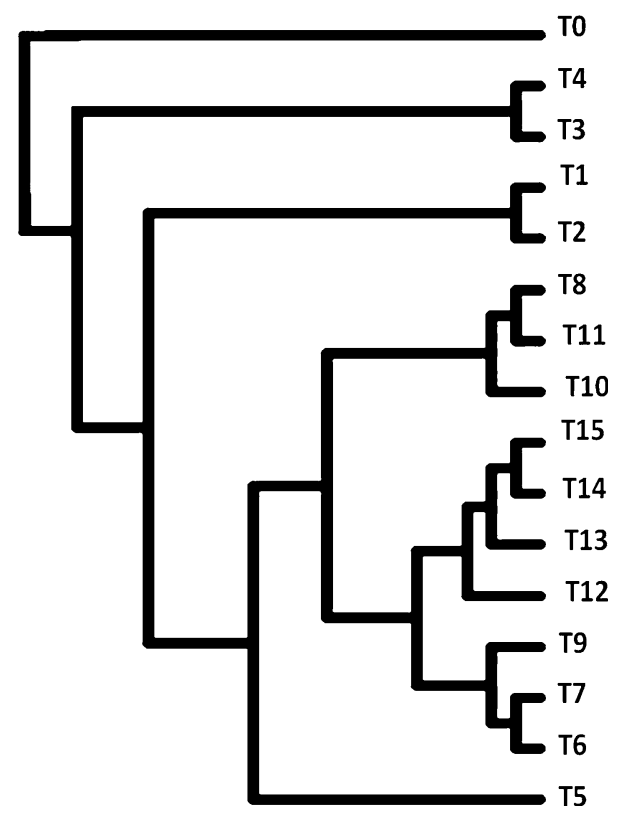

Fig. 2 Dendrogram obtained from hierarchical clustering analysis of the ARDRA band profiles. The calculation was performed using the PHYLIP (Phylogeny Interference Package, version 3.6 Dept of Genome Sciences, University of Washington, Seattle, http://evolution. genetics.washington.edu/phylip/getme.html). The sample's ID (T0-T15) are summarized in Table 1

acute enterocolitis episode, in agreement with the use of metronidazole before the onset of these events.

It is noteworthy that the microflora profiles in partial (T3, T14) and total remission (T4, T15) were always more similar than the enterocolitis ones and were associated with specific antibiotic treatment. The remission in all cases was associated with the suspension of metronidazole treatment and the use of amoxicillin/clavulanic acid resolved the issue. The effect of change in antibiotic regimen on the microflora can be appreciated also in the short term: the restriction profiles of sample T9 was different from that of sample T10 and T11, following 6 and $12 \mathrm{~h}$ of doubling the dose of amoxicillin/clavulanic acid, respectively. Some of the strains isolated during enterocolitis or remission phases were also analyzed using ARDRA on single colonies than on the pool. The results obtained on cultivated strains were not similar to those of the pools and the analysis of pure cultures was not able to discriminate enterocolitis from remission (data not shown).

\section{Discussion}

In Hirschsprung's disease-associated enterocolitis, fecal bacteria concentrations increase at different levels with severity of disease. The alteration in number and localization of bacteria can be the result of colon resection. In particular, the anal sphincter hypertone resulting from resection and ileo-anal anastomosis in long-segment HSCR can cause retention and overgrowth of bacterial intestinal biomass, both in the lumen and deep within the mucosa (entero-adherent bacteria). In patients who underwent resection of the ileo-cecal valve, a number of bacterial species typically present in the colon can be found also in the ileum. It is possible, therefore, to hypothesize a pathogenic role of the normal flora as a result of disruption of components in the immune system or lack of proper training of the immune systems cells in the Peyer's patches, an organ of which tyrosine kinase receptor RET is a key regulator [25].

Despite the recent advances in HSCR treatment, the precise role of intestinal bacteria remains elusive. So far, all the cultivation-based studies investigating bacterial composition of HSCR patients have been hampered by the fact that less than $30 \%$ of total microflora can be cultured. Studies in healthy subjects based on the analysis of $16 \mathrm{~S}$ rRNA gene have been able to characterize approximately $90 \%$ of the dominant fecal microflora [26]. ARDRA of $16 \mathrm{~S}$ rDNA is commonly used to identify major groups of bacterial species. In this study, we evaluated the applicability of ARDRA analysis on DNA extracted directly from fresh feces in HSCR patients with enterocolitis and whether the results reflect the variation in composition of bacterial species associated with enterocolitis.

We demonstrated the utility and power of ARDRA analysis on pools of genomic DNA extracted from stools in assessing microflora dynamics, indicating that the differences in the microflora associated with disease and remission in this specific case are likely to result from different antibiotic therapies. Our findings indicate that rather than due to the appearance of a specific bacterial pathogen, enterocolitis seems to result from a change in the equilibrium between bacterial species as shown in hierarchical clustering analysis of the ARDRA band profiles (Fig. 2). The fact that two of the three samples of the same episode, e.g. T6 and T7, cluster together while the third (T8) clusters apart indicates that the clustering is driven by drastic changes in the microbial pattern induced by the change in antibiotic regimen, rather than time. The onset of enterocolitis is clearly associated with a similar pattern of microbial species as explained by the observation that preenterocolitis sample T9 clusters close to acute enterocolitis $\mathrm{T} 7$ and pre-enterocolitis T6, while remission of the first episode T8 clusters close to antibiotic-associated preremission of another episode, T10 and T11. The inability to capture transient changes suggest that the coarseness of the sampling is not fine enough. Additional time points, in particular in the transition stage, would be needed to capture the details of a rapid transition in the composition of the microbial communities. 
Additionally, we showed that the differences between enterocolitis and remission cannot be appreciated using cultivation techniques and isolation in pure culture. The concept of condition-dependent pathogenic role of microflora, studied mainly in Crohn's disease, has been termed dysbiosis [27] and could probably be generalized also to HSCR with enterocolitis.

The technology used does not identify the culprit, but indeed can discriminate the restriction profile associated with the disease. Recently, Palmer et al. [28] developed a gut-specific DNA microarray containing 8,681 sequences of $16 \mathrm{~S}$ rDNA representing 359 different species of bacteria, Archea and Eucharia. In particular, they tested and optimized a DNA oligonucleotide microarray composed of 10.462 small subunit (SSU) ribosomal DNA (rDNA) probes (8,681 unique sequences) selected to provide quantitative information on the taxonomic composition of diverse microbial populations. The authors affirmed that the estimates of bacterial species abundance obtained using this microarray are similar to those obtained by phylogenetic analysis of SSU rDNA sequences from the same samples, which is considered the "gold standard" method for profiling microbial communities. The limitation of these techniques is that these are time consuming and expensive, in particular requiring highly sophisticated and intensive bioinformatics analyses. It is therefore extremely important to select the most informative samples for these highly sophisticated analyses. These results show the potential of ARDRA as a rapid inexpensive tool to assess variations in the microflora dynamics during HAEC episodes. Understanding the actual determinants of HAEC will require the application of sequence-based metagenomic approaches enabling identification of species associated with health and disease, in a much larger number of HAEC patients. In this perspective, ARDRA could be useful in screening large numbers of samples to select the most informative one for further $16 \mathrm{~S}$ rDNA sequencing analysis.

\section{Conclusion}

We demonstrated that ARDRA is a rapid and inexpensive tool to follow microflora dynamics during Hirschsprung's enterocolitis. Enterocolitis in this patient proved to be dependent on the type of antibiotic treatment with subsequent alteration of the microbial equilibrium resulting in a change in the overall species composition. The unbalance can be recovered by high doses of antibiotic therapy. The conclusions drawn derive from a mixed sample, but identifying the bacterial species associated with the enterocolitis is beyond the scope of this experiment. Yet, the monitoring of the microflora allows discriminating the different samples and following the enterocolitis in a dynamic way. ARDRA can be used to select the most informative samples for more detailed analyses and also as a monitoring approach to assess the probability of onset of enterocolitis and the efficacy of a specific antibiotic regimen.

\section{References}

1. Pini Prato A, Avanzini S, Gentilino V et al (2007) Rectal suction biopsy in the workup of childhood chronic constipation: indications and diagnostic value. Pediatr Surg Int 23:117-122

2. Pini Prato A, Gentilino V, Giunta C et al (2008) Hirschsprung's disease: 13 years' experience in 112 patients from a single institution. Pediatr Surg Int 24:175-182

3. Suita S, Taguchi T, Ieiri S et al (2005) Hirschsprung's disease in Japan: analysis of 3852 patients based on a nationwide survey in 30 years. J Pediatr Surg 40:197-201 discussion 201-202

4. Elhalaby EA, Teitelbaum DH, Coran AG et al (1995) Enterocolitis associated with Hirschsprung's disease: a clinical histopathological correlative study. J Pediatr Surg 30:1023-1026 discussion 1026-1027

5. Murphy F, Puri P (2005) Clostridium difficile-associated diarrhea in 200 Canadian children. Pediatr Surg Int 21:773-779

6. Moore SW, Sidler D, Zaahl MG (2008) The ITGB2 immunomodulatory gene (CD18), enterocolitis, and Hirschsprung's disease. J Pediatr Surg 43:1439-1444

7. Strober W, Fuss I, Mannon P (2007) The fundamental basis of inflammatory bowel disease. J Clin Invest 117:514-521

8. Chinen H, Matsuoka K, Sato T et al (2007) Lamina propria c-kit+ immune precursors reside in human adult intestine and differentiate into natural killer cells. Gastroenterology 133:559-573

9. Luci C, Reynders A, Ivanov II et al (2009) Influence of the transcription factor RORgammat on the development of NKp46+ cell populations in gut and skin. Nat Immunol 10:75-82

10. Satoh-Takayama N, Vosshenrich CA, Lesjean-Pottier S et al (2008) Microbial flora drives interleukin 22 production in intestinal NKp46+ cells that provide innate mucosal immune defense. Immunity 29:958-970

11. Morinville V, McDonald J (2005) Clostridium difficile-associated diarrhea in 200 Canadian children. Can J Gastroenterol 19:497501

12. Chong PP, Chieng DC, Low LY et al (2006) Recurrent candidaemia in a neonate with Hirschsprung's disease: fluconazole resistance and genetic relatedness of eight Candida tropicalis isolates. J Med Microbiol 55:423-428

13. Eckburg PB, Bik EM, Bernstein CN et al (2005) Diversity of the human intestinal microbial flora. Science 308:1635-1638

14. Muyzer G, Smalla K (1998) Application of denaturing gradient gel electrophoresis (DGGE) and temperature gradient gel electrophoresis (TGGE) in microbial ecology. Antonie Van Leeuwenhoek 73:127-141

15. Chèneby D, Philippot L, Hartmann A et al (2000) 16S rDNA analysis for characterization of denitrifying bacteria isolated from three agricultural soils. FEMS Microbiol Ecol 34:121-128

16. Cho JC, Tiedje JM (2000) Biogeography and degree of endemicity of fluorescent Pseudomonas strains in soil. Appl Environ Microbiol 66:5448-5456

17. Zoetendal EG, Heilig HG, Klaassens ES et al (2006) Isolation of DNA from bacterial samples of the human gastrointestinal tract. Nat Protoc 1:870-873 
18. Sancandi S, Ceccherini I, Costa M et al (2000) Population-based study of the incidence of RET mutations in Hirschsprung's disease patients. J Pediatr Surg 35:139-142

19. Lantieri F, Griseri P, Puppo F et al (2006) Haplotypes of the human RET proto-oncogene associated with Hirschsprung disease in the Italian population derived from a single ancestral combination of alleles. Ann Hum Genet 70:12-26

20. Emison ES, McCallion AS, Kashuk CS et al (2005) A common sex-dependent mutation in a RET enhancer underlies Hirschsprung disease risk. Nature 434:857-863

21. Koeleman JG, Stoof J, Biesmans DJ et al (1998) Comparison of amplified ribosomal DNA restriction analysis, random amplified polymorphic DNA analysis, and amplified fragment length polymorphism fingerprinting for identification of Acinetobacter genomic species and typing of Acinetobacter baumannii. J Clin Microbiol 36:2522-2529

22. Vaneechoutte M, Riegel P, de Briel D et al (1995) Evaluation of the applicability of amplified rDNA-restriction analysis (ARDRA) to identification of species of the genus Corynebacterium. Res Microbiol 146(8):633-641
23. Vaneechoutte M, De Beenhouwer H, Claeys G et al (1993) Identification of Mycobacterium species by using amplified ribosomal DNA restriction analysis. J Clin Microbiol 31(8): 2061-2065

24. Hendy MD, Penny D (1982) Branch and bound algorithms to determine minimal evolutionary trees. Math Biosci 59:277-290

25. Veiga-Fernandes H, Coles MC, Foster KE et al (2007) Tyrosine kinase receptor RET is a key regulator of Peyer's patch organogenesis. Nature 446:547-551

26. Gill SR, Pop M, Deboy RT et al (2006) Metagenomic analysis of the human distal gut microbiome. Science 312:1355-1359

27. Tamboli CP, Neut C, Desreumaux P et al (2004) Dysbiosis in inflammatory bowel disease. Gut 53:1-4

28. Palmer C, Bik EM, Digiulio DB et al (2007) Development of the human infant intestinal microbiota. PLoS Biol 5:e177

29. Pastor AC, Osman F, Teitelbaum DH, Caty MG, Langer JC (2009) Development of a standardized definition for Hirschsprung's-associated enterocolitis: a Delphi analysis. J Pediatr Surg 44(1):251-256 